\title{
miR-145 plays a role in mitochondria dysfunction in alveolar epithelial cells in LPS-induced ARDS
}

\author{
Yi Han \\ Zhongshan Hospital Fudan University

\section{Sucheng Mu} \\ Zhongshan Hospital Fudan University \\ Jianli Wang \\ Zhongshan Hospital Fudan University \\ Wei Wei \\ Zhongshan Hospital Fudan University \\ Ming Zhu \\ Zhongshan Hospital Fudan University \\ Zhenju Song \\ Zhongshan Hospital Fudan University \\ Chaoyang Tong ( $\nabla$ mickie@live.cn ) \\ https://orcid.org/0000-0001-7095-9696
}

\section{Research article}

Keywords: microRNA-145, Mitochondria function, LPS, ARDS

Posted Date: July 25th, 2019

DOl: https://doi.org/10.21203/rs.2.11952/v1

License: (c) (i) This work is licensed under a Creative Commons Attribution 4.0 International License.

Read Full License 


\section{Abstract}

Backgrounds: Acute respiratory distress syndrome (ARDS) causes substantial mortality worldwide. Alveolar epithelium is one of the main sites of cell injury in ARDS. MicroRNAs (miRNAs) are small noncoding RNA molecules that are recognized as endogenous physiological regulators of gene expression. As an important miRNA, miR-145 has been studied in various diseases, while its role in ARDS remains not investigated. Methods: Intratracheal instillation of LPS was used to establish ARDS model. Cytokines from bronchoalveolar lavage fluid (BALF), lung wet/dry ratio as well as the pathological structures using H\&E staining and Transmission electron microscope (TEM) was evaluated; lung miR-145 mRNA expression was detected using qPCR. And further bioinformatics was focused on the target genes and possible pathways of the gene regulation. Results: A rat model of LPS-induced ARDS was well established by intratracheal instillation of LPS. miR-145 was down-regulated in LPS-induced ARDS lung, and mitochondria dysfunction was observed in alveolar epithelial cells in ARDS lung, most obviously at $72 \mathrm{~h}$ after LPS. TargetScan and MirDB were used to predict the target genes of miR-145, a total of 428 overlapping genes were identified, among which, 7 genes were associated with mitochondria function. The KEGG pathways were significantly enriched in MAPK signaling pathway and RAS signaling pathway, and the $\mathrm{GO}$ biological process terms were mainly enriched in gene binding, signal transduction, and regulation of transcription. Conclusions: The current work provided evidence between miR-145 and LPSinduced ARDS. Clearly, miR-145 was down-regulated in LPS induced lung injury, and resultant had impact on its downstream genes targeting mitochondria functions through MAPK and RAS signaling pathway.

\section{Background}

Acute respiratory distress syndrome (ARDS) is a very common condition associated with critically ills, which causes substantial mortality worldwide. Approximately 200,000 ARDS cases per year occurs in the U.S. and the mortality is as high as $36-44 \%$

[1-2]. Sepsis is often the major cause of ARDS that may lead to multiple organ failure [3].

Lung inflammation, hypoxemia and non-cardiogenic pulmonary edema formation are the characteristic features [4-5].

Alveolar epithelium is one of the main sites of cell injury in ARDS. Neutrophils contribute to lung inflammation and play important roles in the pathogenesis and progression of ARDS. The activated neutrophils damage epithelial cells [6], which causes increased entry of fluid into the alveolar lumens, decreased clearance of fluid from the alveolar airspace, and decreased production of surfactant [7].

MicroRNAs (miRNAs) are small (19-23 nucleotides) noncoding RNA molecules that are currently being recognized as endogenous physiological regulators of gene expression [8]. Given an individual miRNA could potentially alter complicated cellular processes including cell growth, apoptosis, inflammatoryimmune responses and cell- cell interaction [9-12], it is not surprising that their wrong settings may be involved in pathogenesis of ARDS. 
Many miRNAs are expressed in the lung. miR-17, miR-92a and $m i R-127$ have been shown to regulate lung development [13-14]. Despite miRNAs play a dominating role in several physiological functions, they also are involved in the pathogenesis of many diseases. miRNAs dysregulations are linked to cardiac disorders [15], vascular diseases

[16], cancer [17], and pulmonary diseases, including ARDS [18].

As an important miRNA, miR-145 has been studied in various cancers [19-21]. It could potentially alter complex cellular processes, such as cell growth, cell cycle, apoptosis and invasion [12], some of which through disruption of the epithelial barrier [22]. Also, previous studies have shown that the expression of miR-145 in the rat heart transplantation-induced ischemic reperfusion(l/R) injury were significantly reduced [23-24], indicating that the abnormal expression of miR-145 was involved in myocardial I/ $R$ injury. And hypoxia could promote umbilical cord mesenchymal stem cells (UCMSCs) differentiation into alveolar epithelial cells, and this effect was mainly mediated by miR-145[25]. Also, LPS induced liver inflammation was probably mediated by miR-145 through IRAK1 and NF-KB pathway [26]. Furthermore, miR-145 suppression reversed the LPS-induced inflammatory injury on ATDC5 cells [27].

However, the role of miR-145 in ARDS still remains not investigated. In the present study, we aimed to identify miRNA-145 involved in ARDS originating from studies using an animal model of ARDS. In addition, we tried to focus on the relationship between miRNA-145 and mitochondria function, which plays a critical role in regulating the cell injury of ARDS.

\section{Methods}

Animals. The animal study was proved by the Ethics Department of Zhongshan Hospital, Fudan University. 24 Male Sprague-Dawley rats, 6-8 weeks old, purchased from the Animal Center of Fudan University, bred under pathogen free conditions were housed separately in a temperature-controlled room with a $12 \mathrm{~h}$ light/ $12 \mathrm{~h}$ dark cycle. Animals were allowed free access to food and water.

Animal treatment. Rats were randomly assigned into two groups, and anesthetized with intraperitoneal injection of avertin $(25 \mathrm{mg} / \mathrm{kg})$ and fixed at a $60^{\circ}$ angle on a table in a supine position. The oropharynx was lifted with forceps, allowing for the direct visualization of the trachea. LPS at a dose of $0.5 \mathrm{mg} / \mathrm{kg}$ (Sigma, U.S.) was injected into the trachea using an $18 \mathrm{G}$ catheter attached to a $1 \mathrm{ml}$ syringe as preciously described [28]. Control animals received equal volume of PBS. Rats were sacrificed at 6, 24 and 72 hours after LPS instillation after intraperitoneal injection of avertin $(25 \mathrm{mg} / \mathrm{kg})$.

RNA isolation and analysis. RNA was isolated from the median and caudal lobe of right lung using the RNeasy Mini Kit (QIAGEN) following the manufacturer's instructions. Real-time PCR was performed for expression of microRNAs using the primers as follows.

Primers for microRNA145: GUCCAGUUUUCCCAGGAAUCCCU 
Determination of Lung Water Content. The right main bronchus was ligated, and cranial and accessory lobe of the right lung were excised. After wet weights measured, cranial and accessory lobe of the right lung were placed in an oven at $60^{\circ} \mathrm{C}$ for $72 \mathrm{~h}$ to allow determination of the wet-to-dry weight ratio.

Bronchoalveolar Lavage. The bronchoalveolar lavage (BAL) was performed in the left lung. $2 \mathrm{ml}$ PBS (4 ${ }^{\circ} \mathrm{C}$ ) was slowly infused, after which the fluid was slowly withdrawn and reinfused for additional two times. The recovered fluid was collected for further analysis.

Cytokines in BALF. TNF-a and IL- 6 levels in BALF were measured using rat TNF- $\alpha$ and IL- 6 ELISA kits (R\&D Systems) according to the manufacturer's recommendations.

H\&E Staining. Lung tissues were fixed and processed for $\mathrm{H} \& \mathrm{E}$ staining. Briefly, lung tissues were fixed by $10 \%$ PBS-buffered formalin through trachea catheterization at a transpulmonary pressure of $15 \mathrm{~cm} \mathrm{H}_{2} \mathrm{O}$, and then overnight at $4^{\circ} \mathrm{C}$ with agitation. After paraffin processing, the tissues were cut into semi-thin 4 to $5 \mu \mathrm{m}$ thick and stained with $\mathrm{H} \& \mathrm{E}$ for histological analysis.

Transmission Electron Microscope. Preparation of lung tissues for transmission electron microscopy was following the procedure described previously [29]. Briefly, Lung samples were obtained and fixed with $2.5 \%$ glutaraldehyde in PBS buffer. Next,

lung samples were post-fixed with $1 \%$ OsO4 in PBS buffer for $1 \mathrm{~h}$, and following dehydration, tissues were embedded in $50 \%$ propylene oxide/ $50 \%$ resin. Sectioning was performed on a ultramicrotome $(60 \mathrm{~nm}$ thickness). Samples were then stained with lead citrate, and examined with an electron microscope (Hitachi H-600, Japan).

Target gene prediction of miRNA. The target genes of prognostic miRNA-145 were predicted using TargetScan and miRDB analysis tools. Then, the overlapping genes were analyzed. Gene Ontology (GO) and Kyoto Encyclopedia of Genes and Genomes (KEGG) pathway enrichment analyses were then performed for the target genes. The $P$ value $<0.05$ and gene count $\geq 3$ were set as the cut-off criteria.

Statistical analysis. The data were expressed as mean \pm standard deviation (SD). The expression levels of miRNAs in ARDS and control rats were analyzed by unpaired $t$ test. $P$ value $<0.05$ was considered as statistically significant. The statistical analysis was performed using SPSS Statistics software and Prism 6.0 .

\section{Results}

\section{Results}


The cytokines in BALF were measured at $6 h(n=4), 24 h(n=4)$, and $72 h(n=4)$ after LPS instillation. And the results showed that TNF-a peaked at $6 \mathrm{~h}$ after LPS and then gradually decreased, and IL-6 peaked at $24 \mathrm{~h}$ after LPS, and gradually go back to baseline at 72h (Fig.1 G-H). Moreover, $6 \mathrm{~h}$ after LPS injection, W/D ratio of LPS lungs was about 7.5 compared with 3.0 in control lungs; $24 \mathrm{~h}$ after LPS injection, W/D ratio of LPS lungs was about 7.0, still much higher than control group; and $72 \mathrm{~h}$ after LPS injection, W/D ratio of LPS lungs was about 4.5, still higher than control group (Fig. $1 \mathrm{~F}$ ). Also, with H\&E staining, there were little inflammatory cell or lymphocytes infiltration, and the structure of alveolar was almost intact in the control group, and 6h after LPS injection, the lymphocytes in the alveolar was increased, especially increased around bronchi and lung vessels, and there was protein-like fluid filled in the alveola. 24h after LPS injection, there were more lymphocytes infiltration, the alveolar structures were disrupted, filled with inflammatory cells, and alveolar interval were much thicker than control; and at $72 \mathrm{~h}$ after LPS injection, there were still lymphocytes infiltration, while a little decrease than 24h (Fig.1 A-D). Using lung injury scoring system ${ }^{[30]}$ (Table 1), there were significantly increase of the scores in LPS instillation lungs, especially at $24 \mathrm{~h}$ and $72 \mathrm{~h}$ after LPS (Fig.1 E).

Fig.1 A-D. Histology of rat lung with and without LPS instillation (H\&E staining

$\times 10)$. Red star: Lymphocytes infiltration; Blue arrow: Protein-like fluid filled in alveola; Black arrow: Alveolar interval thickened. A. Control lung; B. 6h after LPS instillation;

1. 24h after LPS instillation; D. 72h after LPS instillation. E. Score of acute lung injury $(\times 100)$. F. W/D ratio of rat lungs in both control and LPS group. G-H. Change of cytokines concentration in BALF.

Table1. Lung injury scoring system ${ }^{[30]}$

\begin{tabular}{|c|c|c|}
\hline \multirow[t]{2}{*}{ Parameters } & \multicolumn{2}{|c|}{ Score per field } \\
\hline & $0 \quad 1$ & 2 \\
\hline A Neutrophils in the alveolar space & None1-5 & $>5$ \\
\hline B Neutrophils in the interstitial space & None 1-5 & $>5$ \\
\hline C Hyaline membranes & None1 & $>1$ \\
\hline D Proteinaceous debris filling the airspaces & None1 & $>1$ \\
\hline E Alveolar septal thickening & $<2 x \quad 2\rangle$ & $x>4 x$ \\
\hline
\end{tabular}

Score $=[(20 \times A)+(14 \times B)+(7 \times C)+(7 \times D)+(2 \times E)] /$ (number of fields $\times 100)$

2. miR-145 was down-regulated in LPS-induced ARDS rat 
miR-145 mRNA expression was measured at $6 \mathrm{~h}, 24 \mathrm{~h}$ and $72 \mathrm{~h}$ after LPS instillation using qPCR, the results showed that with LPS instillation, the miR-145 expression was significantly decreased, and there was a more than 10-fold decreasing at 72h after LPS instillation (Fig.2 A-C).

Fig.2 A-C. Rat lung miRNA145 expression with LPS instillation. miRNA 145 was down-regulated at 6h, $24 \mathrm{~h}$ and $72 \mathrm{~h}$ after LPS.

3. Mitochondria dysfunction was found in LPS-induced ARDS rat lung epithelial

The supermicro structure of alveolar epithelial cells were detected using transmission electron microscope. As it showed, there were clear nuclear and lamellar body in control lung epithelial cells, and the electron density of mitochondria was homogeneous. At $6 \mathrm{~h}$ after LPS, the villus of epithelial cell turned incomplete, while there still were intact lamellar body and mitochondria. At 24h after LPS, the lamellar body and mitochondria cristae structure were in disorder, and the electron density of mitochondria was gradually higher. At $72 \mathrm{~h}$ after LPS, there were obvious structure disturbance of mitochondria and lamellar body, with absence of mitochondrial cristae, which were obvious evidences of mitochondria injury (Fig.3 A-D).

Fig.3 TEM structure of alveolar epithelial cell $(\times 20000)$. Red star: Nuclear of alveolar epithelial cell; Blue arrow: Lamellar body; Green arrow: Mitochondria; Black arrow: Villus of alveolar epithelial cell. A. Control lung; B. 6h after LPS instillation; C. 24h after LPS instillation; D. 72h after LPS instillation.

4. Predicted target genes of miR-145 was related to mitochondria

TargetScan and MirDB databases were used to do the searching of miR-145, as to predict the target genes, and a total of 428 overlapping genes were identified (Fig.4 A). Then, enrichment analysis was performed to elucidate the biological function of consensus target genes. The KEGG pathways were significantly enriched in MAPK signaling pathway and RAS signaling pathway. In addition, the GO 
biological process terms were mainly enriched in gene binding, signal transduction, and regulation of transcription (Fig.4 D-E). Moreover, using rat genome database with NCBI database, seven mitochondria associated genes regulated by miR-145 were identified, including Slc1a2, Cftr, Ogt, Acs14, Camk2d, Slc8a3 and Slc25a25 (Fig.4 B-C).

Fig.4 Bioinformatics data of miR-145 target genes and pathways. A.Target genes of miR145 predicted using TargetScan, and MirDB tools. B,C. Mitochondria associated genes regulated by miR145. D. KEGG pathways analysis. E. GO biological process.

\section{Discussion}

ARDS is one of the most critical disease in ICU, which seriously affects the prognosis and quality life of critically ill patients. It is characterized by acute onset of respiratory failure associated with diffuse interstitial pulmonary edema in the absence of left ventricular failure. It has been proved that the degeneration of surfactant is one of the most important cause of ARDS. Reduced secretion of surfactant is associated with worse outcomes [31-33]. A potential role for intact mitochondria in surfactant production and secretion are supported by studies reporting that intramitochondrial delivery of glutathione in rats significantly preserved surfactant-producing and secreting function of type II cells [34].

Plenty of evidences exhibited that LPS could induce cells inflammatory responses in various diseases, of course including ARDS [26-28]. Instillation of LPS intratracheal is proved to be an excellent in vivo model of lung injury and it is widely used for investigating ARDS. In our study, the results revealed that LPS upregulated IL -6 and TNF-a expressions, promptly stimulated cytokines responses, and significantly damaged the epithelial barrier which lead to increased intracellular water content. And a mass of inflammatory cells infiltrated in the alveolar air space as well as the para-vascular space, the separation of alveola was much thicken after LPS instillation, which would finally form the hyaloid membrane in ARDS. With these pathological manifestations, we scored the inflammatory and structure disruption level based on the

previous study [30], and we concluded that intratracheal instillation of LPS is a convenient and sufficient way to set up an ARDS model in rat.

MicroRNAs are small non-coding RNA that plays a crucial role in many disease processes, including malignancy and inflammatory processes. Abnormal expression miRNAs, such as miR-126-5p, miR$1246, \mathrm{miR}-34 \mathrm{a}, \mathrm{miR}-27 \mathrm{a}$ and mir-223 have been found in lung injury [28,35-38]. MiR-145 is an important molecular marker, which has been proven to mediate cell proliferation, cell cycle, apoptosis and 
invasion [19]. Researchers also found miR-145 played a role in cervical epithelial cell barrier [22]. These studies demonstrated that miR-145 was implicated in epithelial cell damage in cancers. However, whether miR-145 was involved in regulating LPS-induced ARDS remain unknown.

In our study, we found that the expression of miR-145 was decreased in ARDS lungs, with the most obviously decreasing at $72 \mathrm{~h}$ after LPS injection, which was corresponding to the level of mitochondria damage observed by TEM in lung epithelial cells. We found the electron density of mitochondria began higher since $24 \mathrm{~h}$ after LPS injection, and there was obvious structure disturbance of mitochondria and lamellar body, as well as with absence of mitochondrial cristae. As described in previous studies, miR145 plays an important role in regulating mitochondrial apoptotic pathway in tumor cells, partly through its ability of targeting various anti-apoptotic molecules [39]. Moreover, the aberrant expression of miR145 has been shown to be associated with vascular smooth muscle cells' response to hydrogen peroxideinduced oxidative stress, indicating that miR-145 may participate in the regulation of the oxidative stress- triggered apoptosis and the regulation of the mitochondrial apoptotic pathway [24]. Furthermore, PDCD4 was identified as a novel target of miR-145 in cardiomyocytes, overexpression of PDCD4 could remarkably restore the miR-145-inhibited cardiomyocytes apoptosis and mitochondrial dysfunction after hypoxia injury [40]. However, little is known about whether miR-145 is associated with lung epithelial cell apoptosis under LPS challenge or how it interferes with the mitochondrial apoptotic pathway.

Therefore, we used databases searching of miR-145, to predict the target genes of miR- 145 in mitochondria, and we found using TargetScan and MirDB analysis tools, a total of 428 overlapping genes were identified. Then, enrichment analysis was performed to elucidate the biological function of consensus target genes. The KEGG pathways were significantly enriched in MAPK signaling pathway and RAS signaling pathway. In addition, the GO biological process terms were mainly enriched in gene binding, signal transduction, and regulation of transcription. Moreover, using rat genome database with NCBI database, seven mitochondria associated genes regulated by miR145 were identified, including Slc1a2, Cftr, Ogt, Acs14, Camk2d, Slc8a3 and Slc25a25. Previous studies have indicated that inherited mitochondrial polymorphisms, genes, and pathways were associated with epithelial ovarian cancer risk, including MTERF, PPARGC1A, and CAMK2D[41]; also, CFTR silencing results in lipid homeostasis

disruption and mitochondrial dysfunction in intestinal epithelial cells, and it regulates neuronal apoptosis following cerebral I/R via mitochondrial oxidative stress-dependent pathway[42-43]; $\mathrm{MOGT}$ is catalytically active in vivo and supports mitochondrial structure, health, and survival[44].

\section{Conclusion}

The current work provided evidence related to the role of miR-145 in mitochondria function in LPSinduced ARDS. Clearly, miR-145 was down-regulated in LPS induced lung injury, and resultant had impact on its downstream genes targeting mitochondria functions through MAPK and RAS signaling pathway. These observations and predictions provided evidence that miR-145 may play a role in 
inflammatory-related epithelial barrier disruption, and further studies are needed to delineate the specific mechanisms.

\section{Abbreviations}

Acute respiratory distress syndrome, ARDS MicroRNAs, miRNAs

Ischemic reperfusion injury, l/R injury

Umbilical cord mesenchymal stem cells, UCMSCs Bronchoalveolar lavage, BAL

Gene Ontology, GO

Kyoto Encyclopedia of Genes and Genomes, KEGG

\section{Declarations}

- Ethics approval and consent to participate

The animal study was approved by the Ethics Department of Zhongshan Hospital, Fudan University.

- Consent for publication Not applicable.

- Availability of data and material

All data generated or analyzed during this study are included in this published article. 4.Competing interests

The authors declare that they have no competing interests. 5.Funding

Not applicable. 6.Authors' contributions

ZJS and CYT conceived the project and conduct the study. YH, SCM, WW, MZ and JLW contributed to data acquisition. $\mathrm{YH}$ analyzed and interpreted the data. $\mathrm{YH}$ was the

major contributor in writing the manuscript. All authors read and approved the final manuscript.

7.Acknowledgements Not applicable.

\section{References}

[1] Rubenfeld GD, Caldwell E, Peabody E, et al. Incidence and outcomes of acute lung injury. N Engl J Med. 2005; 353(16):1685-1693.

[2] Phua J, Badia JR, Adhikari NK, et al. Has mortality from acute respiratory distress syndrome decreased over time? A systematic review. Am J Res Crit Care Med. 2009; 179:220-227. 
[3] Ware LB, Matthay MA. The acute respiratory distress syndrome. N Engl J Med. 2000; 342:13341349.

[4] Matthay MA, Zemans RL. The acute respiratory distress syndrome: Pathogenesis and treatment. Annu Rev Pathol. 2011; 6:147-163.

[5] Cepkova M, Matthay MA. Pharmacotherapy of acute lung injury and the acute respiratory distress syndrome. J Intensive Care Med. 2006; 21:119-143.

[6] Grommes J, Soehnlein 0: Contribution of neutrophils to acute lung injury. Mol Med. 2011; 17(34):293-307.

[7] Lowe K, Alvarez D, King J, Stevens T. Phenotypic heterogeneity in lung capillary and extra-alveolar endothelial cells. Increased extra-alveolar endothelial permeability is sufficient to decrease compliance. $J$ Surg Res. 2007; 143(1):70-77.

[8] Bartel DP. MicroRNAs: genomics, biogenesis, mechanism, and function. Cell. 2004;116: 281-297.

[9] Bueno MJ, Malumbres M. MicroRNAs and the cell cycle. Biochim Biophys Acta. 2011; 1812:592601.

[10] Chen CZ, Li L, Lodish HF, Bartel DP. MicroRNAs modulate hematopoietic lineage differentiation. Science. 2004; 303:83-86.

[11] Hagen JW, Lai EC. MicroRNA control of cell-cell signaling during development and disease. Cell Cycle. 2008; 7:2327-2332.

[12] Su'e Chang, Ling Gao, Yang Yang, et al. miR-145 mediates the antiproliferative and gene regulatory effects of vitamin D3 by directly targeting E2F3 in gastric cancer cells. Oncotarget. 2015; 6(10):76757685 .

[13] Carraro G, El-Hashash A, Guidolin D, et al. miR-17 family of microRNAs controls FGF10-mediated embryonic lung epithelial branching morphogenesis through MAPK14 and STAT3 regulation of ECadherin distribution. Dev Biol. 2009; 333(2):238-250.

[14] Bhaskaran $M$, Wang $Y$, Zhang $H$, et al. MicroRNA-127 modulates fetal lung development. Physiol Genomics. 2009;37(3):268-278.

[15] Quiat D, Olson EN. MicroRNAs in cardiovascular disease: From pathogenesis to prevention and treatment. J Clin Invest. 2013; 123:11-18.

[16] Zampetaki A, Mayr M. MicroRNAs in vascular and metabolic disease. Circ Res. 2012; 110:508-522. 
[18] Cardinal-Fernandez P, Ferruelo A, Esteban A, Lorente JA. Characteristics of microRNAs and their potential relevance for the diagnosis and therapy of the acute respiratory distress syndrome: From bench to bedside. Transl Res. 2016; 169:102-11.

[19] R.-K. WANG, X.-M. SHAO, J.-P. YANG, et al. MicroRNA-145 inhibits proliferation and promotes apoptosis of HepG2 cells by targeting ROCK1 through the ROCK1/NF-KB signaling pathway. European Review for Medical and Pharmacological Sciences. 2019; 23: 2777-2785.

[20] Victoria J. Findlay, Cindy Wang, Lourdes M. Nogueira, et al. SNAl2 modulates colorectal cancer 5fluorouracil sensitivity through miR145 repression Mol Cancer Ther. 2014; 13(11): 2713-2726

[21] Nicolo Riggi, Mario-Luca Suva, Claudio De Vito. EWS-FLI-1 modulates miRNA145 and SOX2 expression to initiate mesenchymal stem cell reprogramming toward Ewing sarcoma cancer stem cells. Gens \& Development. 2010; 24:916-932.

[22] Lauren Anton, Ann DeVine, Luz-Jeannette Sierra, et al. miR-143 and miR-145 disrupt the cervical epithelial barrier through dysregulation of cell adhesion, apoptosis and proliferation Scientific Reports. 2017; 7: 3020.

[23] Zhao ZH, Hao W, Meng QT, et al. Long non-coding RNA MALAT1 functions as a mediator in cardioprotective effects of fentanyl in myocardial ischemia-reperfusion injury. Cell Biol Int. 2017; 41: 6270.

[24] Li R, Yan G, Li Q, et al. MicroRNA-145 protects cardiomyocytes against hydrogen peroxide-induced apoptosis through targeting the mitochondria apoptotic pathway. PLoS One. 2012; 7: e44907.

[25] Yang Lia, Xu Shi, Liming Yang, et al. Hypoxia promotes the skewed differentiation of umbilical cord mesenchymal stem cells toward type II alveolar epithelial cells by regulating microRNA-145. Gene. $2017 ; 630: 68-75$.

[26] RuiJin Xie, Mei Liu, ShuJie Li. Emodin weakens liver inflammatory injury triggered by lipopolysaccharide through elevating microRNA-145 in vitro and in vivo. Artificial Cells, Nanomedicine, and Biotechnology. 2019; 47(1):1877-1887.

[27] Meihan Liu, Jingzhe Zhang, Wanguo Liu, Wenjun Wang. Salidroside protects ATDC5 cells against lipopolysaccharide-induced injury T through up-regulation of microRNA-145 in osteoarthritis. International Immunopharmacology. 2019; 67:441-448.

[28] MinJie Ju, BoFei Liu, HongYu He, et al. MicroRNA-27a alleviates LPS-induced acute lung injury in mice via inhibiting inflammation and apoptosis through modulating TLR4/MyD88/NF-KB pathway. Cell Cycle. 2018; 17(16):2001-2018. 
[29] Lesley Graham, Jan Marc Orenstein. Processing tissue and cells for transmission electron microscopy in diagnostic pathology and research. Nature Protocols. 2007;2(10): 2439-2450.

[30] Gustavo Matute-Bello, Gregory Downey, et al. An Official American Thoracic Society Workshop Report: Features and Measurements of Experimental Acute Lung Injury in Animals. Am J Respir Cell Mol Biol. 2011; 44:725-738.

[31] Hallman M, Maasilta P, Sipila I, Tahvanainen J. Composition and function of pulmonary surfactant in adult respiratory distress syndrome. Eur Respir J Suppl. 1989; 3:104s-8s.

[32] Kiefmann M, Tank S, Keller P, et al. IDH3 mediates apoptosis of alveolar epithelial cells type 2 due to mitochondrial $\mathrm{Ca}(2+)$ uptake during hypocapnia. Cell Death Dis. 2017; 8:e3005.

[33] Shepard Jr JW, Hauer D, Miyai K, Moser KM. Lamellar body depletion in dogs undergoing pulmonary artery occlusion. J Clin Invest. 1980; 66:36-42.

[34] Guidot DM, Brown LA. Mitochondrial glutathione replacement restores surfactant synthesis and secretion in alveolar epithelial cells of ethanol-fed rats. Alcohol Clin Exp Res. 2000; 24:1070-6.

[35] Yue Fang, Fengying Gao, Jing Hao, Zhenwei Liu. microRNA-1246 mediates lipopolysaccharideinduced pulmonary endothelial cell apoptosis and acute lung injury by targeting angiotensin-converting enzyme 2. Am J Transl Res. 2017;9(3):1287-1296.

[36] Rurong Tang, Ling Pei, Tao Bai. Junke Wang. Down-regulation of microRNA-126-5p contributes to overexpression of VEGFA in lipopolysaccharide-induced acute lung injury. Biotechnol Lett. 2016; 38:12771284.

[37] Shwetha K. Shetty, Nivedita Tiwari, Amarnath S. Marudamuthu, et al. p53 and miR-34a Feedback Promotes Lung Epithelial Injury and Pulmonary Fibrosis. The American Journal of Pathology. 2017; 187(5):1016-1034.

[38] Viola Neudecker, Kelley S. Brodsky, Eric T. Clambey, et al. Neutrophil transfer of miR-223 to lung epithelial cells dampens acute lung injury in mice. Sci Transl Med. 2017; 9(408): 5360.

[39] Junbo Zhou, Jian Gong, Chun Ding, Guiqin Chen. Quercetin induces the apoptosis of human ovarian carcinoma cells by upregulating the expression of microRNA-145. Molecular Medicine Reports. 2015;12: 3127-3131.

[40] Hao Xu, Heng Cao, Guoqing Zhu, et al. Overexpression of microRNA-145 protects against rat myocardial infarction through targeting PDCD4. Am J Transl Res. 2017;9(11):5003-5011. 
[41] Jennifer Permuth-Wey, Y. Ann Chen, Ya-Yu Tsai, et al. Inherited Variants in Mitochondrial Biogenesis Genes May Influence Epithelial Ovarian Cancer Risk. Cancer Epidemiol Biomarkers Prev. 2011; 20(6): 1131-1145.

[42] Marie L. Kleme, Alain Sané, Carole Garofalo, et al. CFTR Deletion Confers Mitochondrial Dysfunction and Disrupts Lipid Homeostasis in Intestinal Epithelial Cells. Nutrients. 2018;10(7):e836.

[43] Ya-Ping Zhang, Yong Zhang, Zhi-Bin Xiao, et al. CFTR prevents neuronal apoptosis following cerebral ischemia reperfusion via regulating mitochondrial oxidative stress. Journal of Molecular Medicine. 2018; 96:611-620.

[44] Juliana L. Sacoman, Raul Y. Dagda, Amanda R. Burnham-Marusich, et al. Mitochondrial O-GlcNAc Transferase (mOGT) Regulates Mitochondrial Structure, Function, and Survival in HeLa Cells. J Biol Chem. 2017; 292(11):4499-4518.

\section{Figures}

A

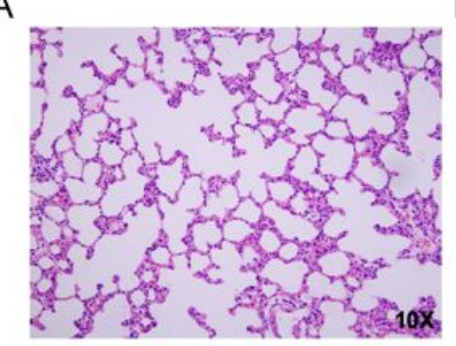

E

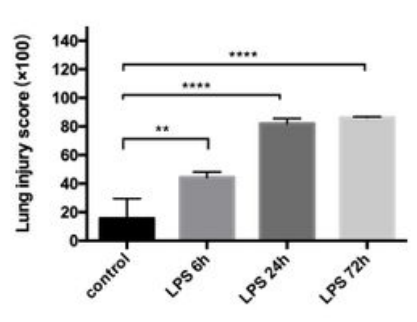

B

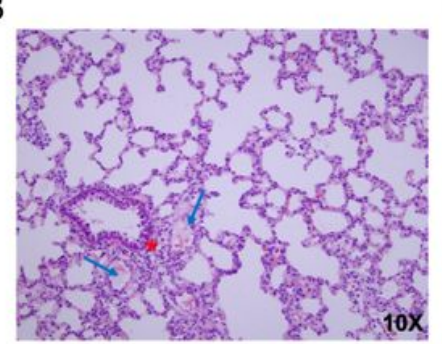

$\mathrm{F}$

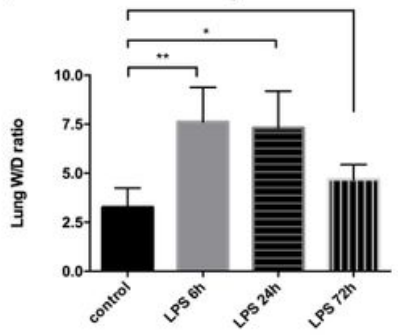

C

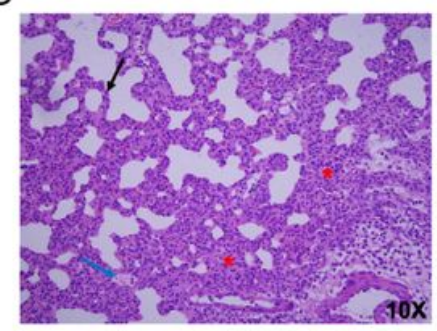

G

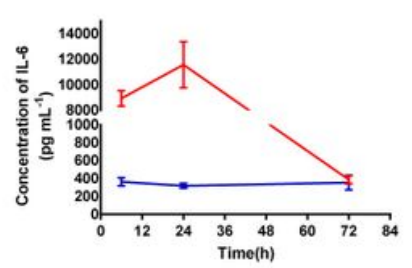

D

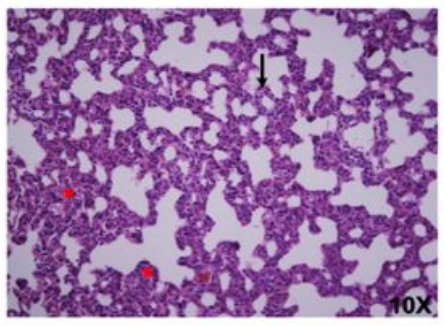

$\mathrm{H}$
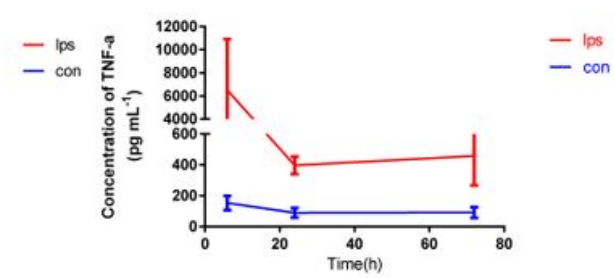

Figure 1

A-D. Histology of rat lung with and without LPS instillation (H\&E staining $\triangle \times 10)$. Red star: Lymphocytes infiltration; Blue arrow: Protein-like fluid filled in alveola; Black arrow: Alveolar interval thickened. A.

Control lung; B. 6h after LPS instillation; C. 24h after LPS instillation; D. 72h after LPS instillation. E. Score of acute lung injury $(\times 100)$. F. W/D ratio of rat lungs 

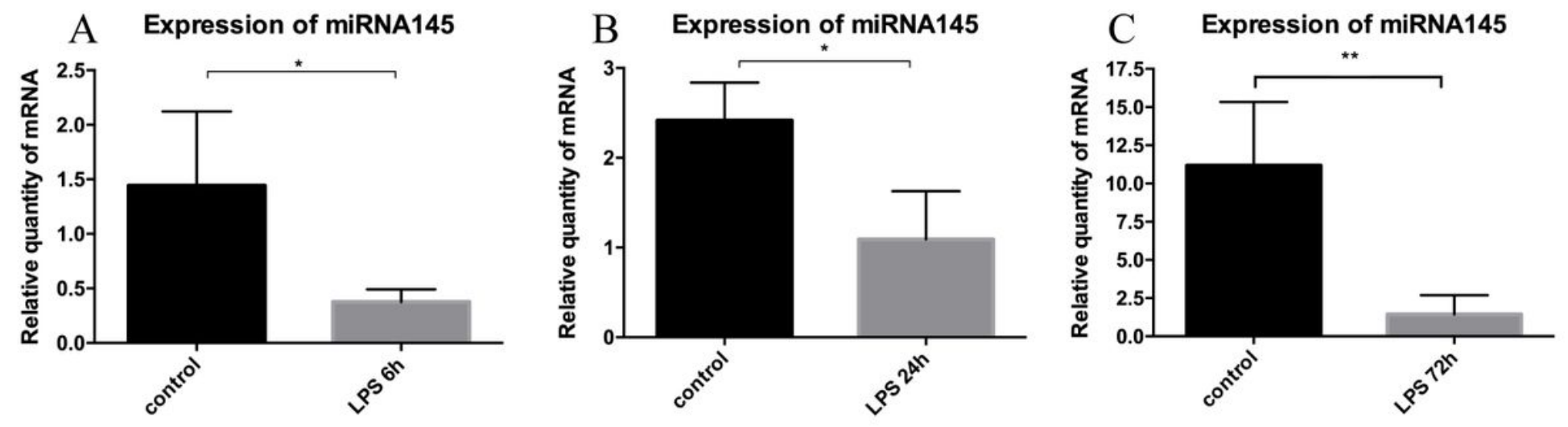

Figure 2

A-C. Rat lung miRNA145 expression with LPS instillation. miRNA 145 was down-regulated at $6 \mathrm{~h}, 24 \mathrm{~h}$ and $72 \mathrm{~h}$ after LPS. 

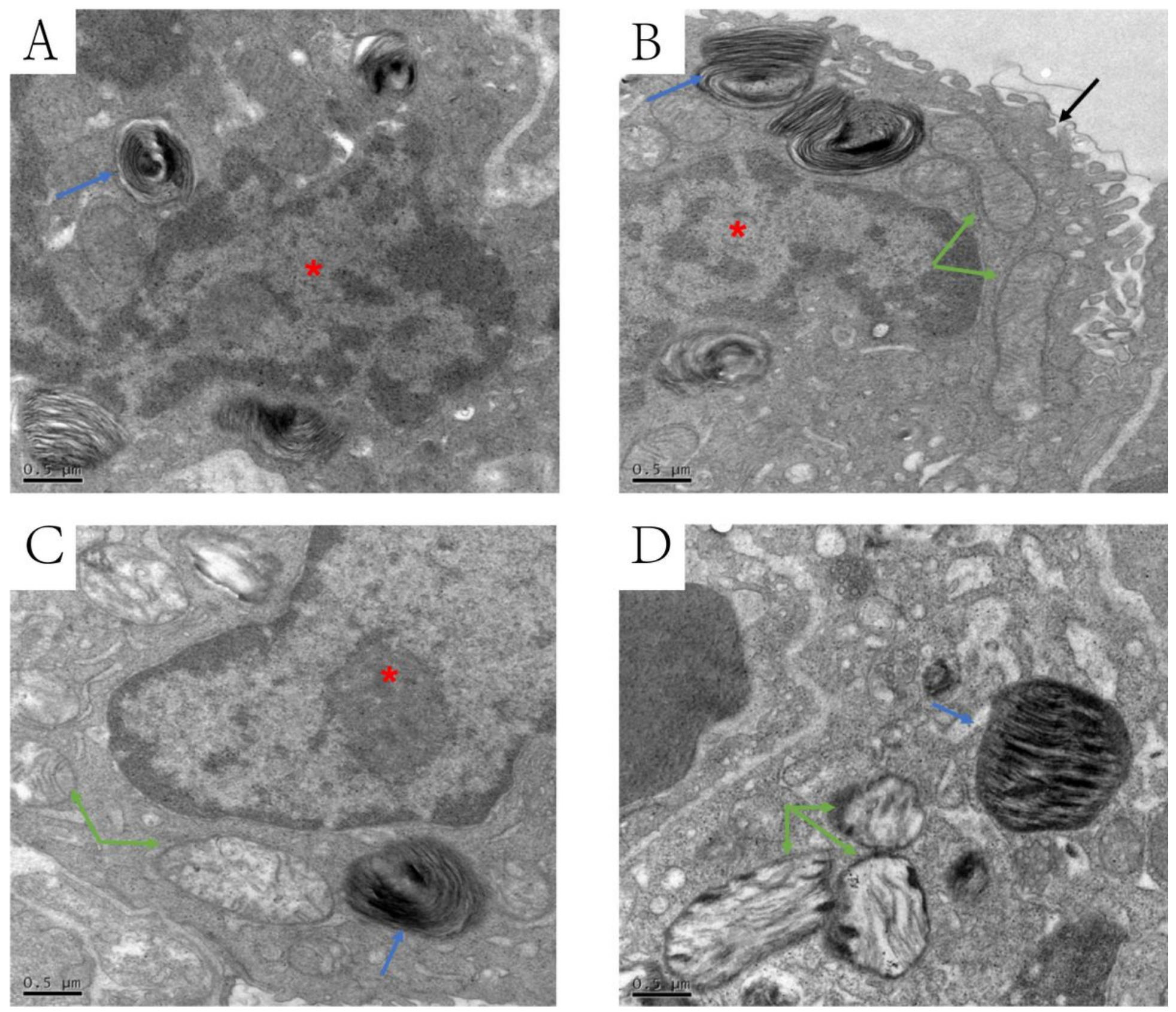

\section{Figure 3}

TEM structure of alveolar epithelial cell $(\times 20000)$. Red star: Nuclear of alveolar epithelial cell; Blue arrow: Lamellar body; Green arrow: Mitochondria; Black arrow: Villus of alveolar epithelial cell. A. Control lung; B. $6 \mathrm{~h}$ after LPS instillation; C. 24h after LPS instillation; D. 72h after LPS instillation. 
A
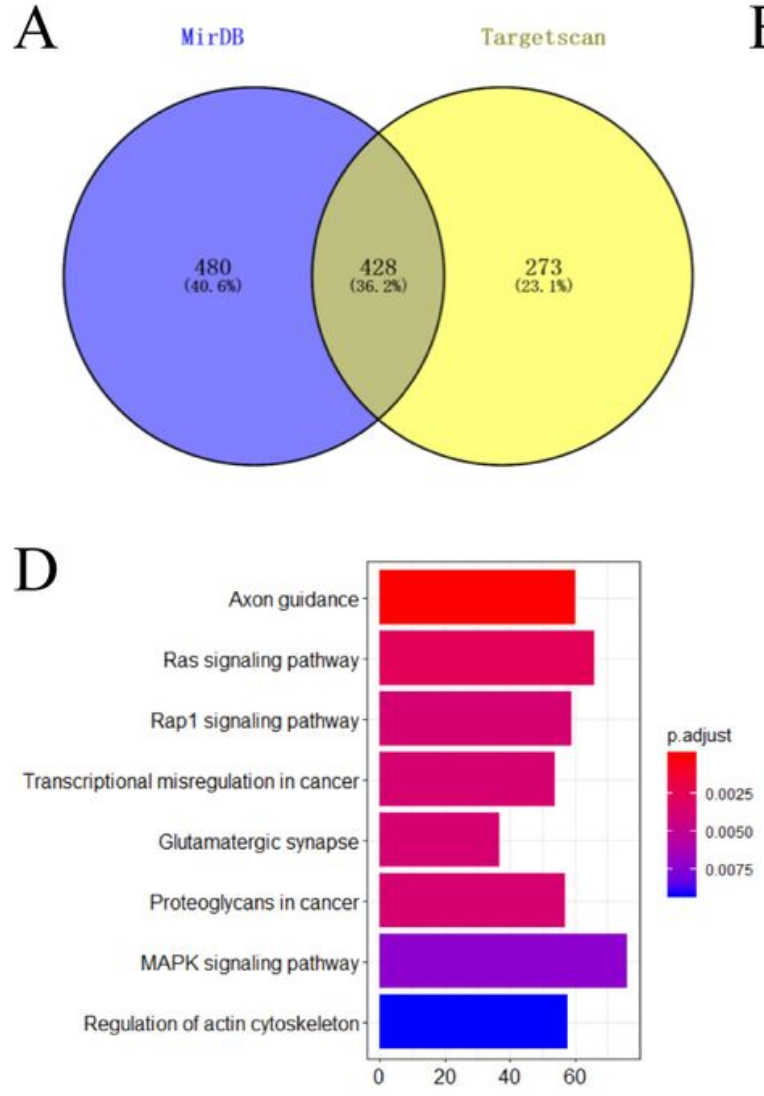

B
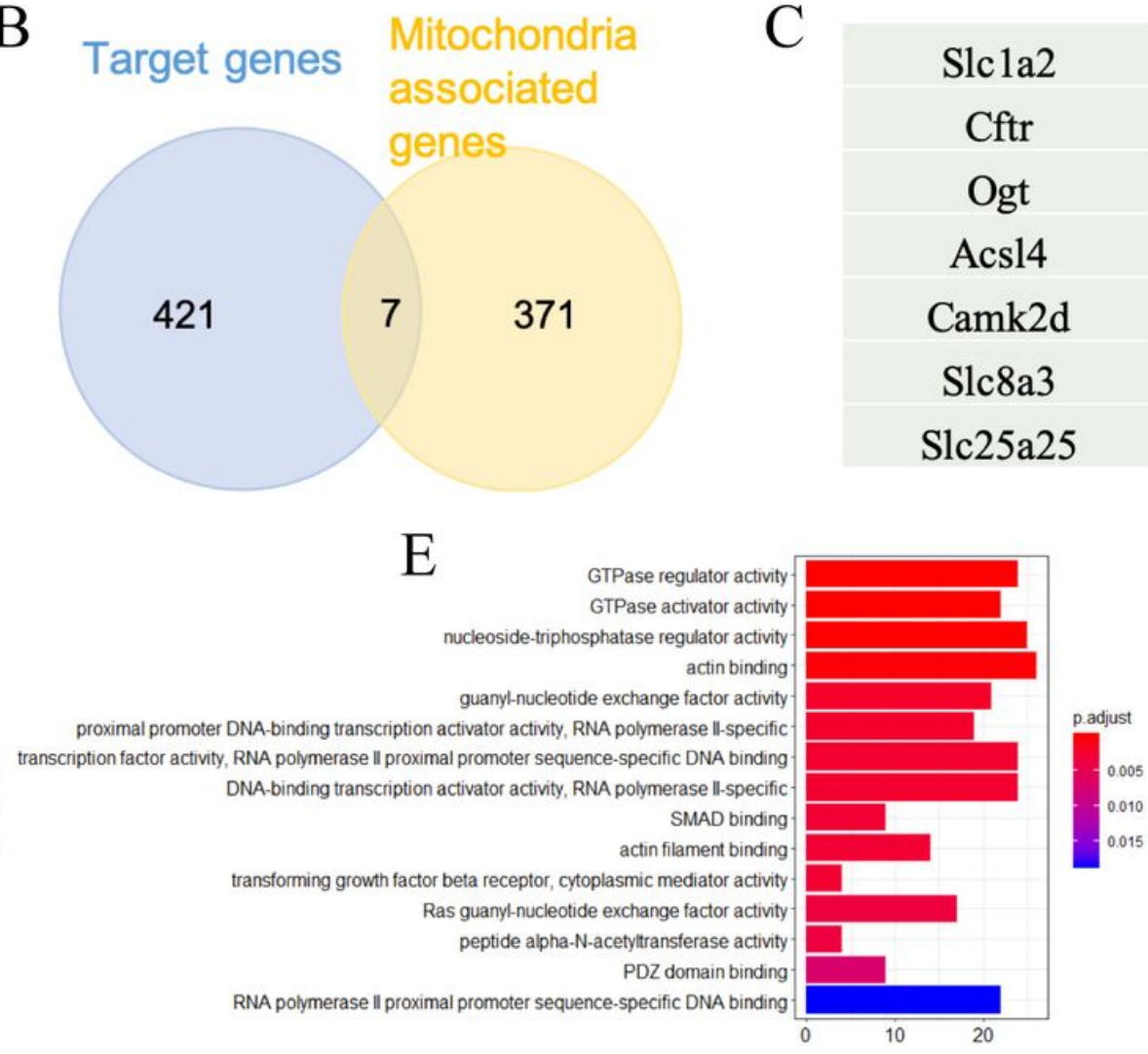

Figure 4

Bioinformatics data of miR-145 target genes and pathways. A.Target genes of miR145 predicted using TargetScan, and MirDB tools. B,C. Mitochondria associated genes regulated by miR145. D. KEGG pathways analysis. E. GO biological process.

\section{Supplementary Files}

This is a list of supplementary files associated with this preprint. Click to download.

- NC3RsARRIVEGuidelinesChecklistfillable.pdf 